\title{
Enhanced Cognitive Radio Resource Management for LTE Systems
}

\author{
Ismail AlQerm*, Basem Shihada*, and Kang G. Shin ${ }^{\dagger}$ \\ * CEMSE Division, KAUST, Saudi Arabia, \{ismail.qerm, basem.shihada\}@kaust.edu.sa \\ $\dagger$ Dept. of Electrical Engineering and Computer Science, University of Michigan, USA, kgshin@eecs.umich.edu
}

\begin{abstract}
The explosive growth in mobile Internet and related services has increased the need for more bandwidth in cellular networks. The Long-Term Evolution (LTE) technology is an attractive solution for operators and subscribers to meet such need since it provides high data rates and scalable bandwidth. Radio Resource Management (RRM) is essential for LTE to provide better communication quality and meet the application QoS requirements. Cognitive resource management is a promising solution for LTE RRM as it improves network efficiency by exploiting radio environment information, intelligent optimization algorithms to configure transmission parameters, and mitigate interference. In this paper, we propose a cognitive resource management scheme to adapt LTE network parameters to the environment conditions. The scheme optimizes resource blocks assignment, modulation selection and bandwidth selection to maximize throughput and minimize interference. The scheme uses constrained optimization for throughput maximization and interference control. It is also enhanced by learning mechanism to reduce the optimization complexity and improve the decisionmaking quality. Our evaluation results show that our scheme achieved significant improvements in throughput and LTE system capacity. Results also show the improvement in the user satisfaction over other techniques in LTE RRM.
\end{abstract}

Index Terms-LTE networks, transmission parameters, decision-making, radio resource management, cognitive radio

\section{INTRODUCTION}

Digital mobile telecommunications have penetrated mass markets with noticeable expansion in data services, increasing the number of mobile users, network providers as well as the heterogeneity of the network. Long-Term Evolution (LTE) [1] promises high data rates, $100 \mathrm{Mbps}$ in the downlink and $50 \mathrm{Mbps}$ in the uplink. It also supports a scalable bandwidth between 1.25 and $20 \mathrm{MHz}$. 3GPP is the standard that manages self-organizing and self-optimizing capabilities of LTE networks. The specification of the Evolved Universal Terrestrial Radio Access (E-UTRA) is still developing through 3GGP [1], [2], [3]. The 3GPP Release 8 LTE has already become a commercial standard and is deployed in many countries. LTE operates in different frequency bands with flexibility to support different bandwidths. In addition, the available spectrum can be easily shared by different types of services. Thus, LTE can provide higher data rate and flexible use of the spectrum. It supports both paired Frequency Division Duplexing (FDD) and unpaired Time Division Duplexing (TDD) band operations. In FDD, the uplink and downlink use different frequency bands. However, they share the same frequency band in case of TDD. Therefore, TDD is better for flexible spectrum usage and different data traffic support. As the goal of LTE is to provide higher data rate with lower latency, Orthogonal Frequency Division Multiple Access (OFDMA) is used as its access technology where radio resources are scheduled in the unit of Resource Blocks (RBs) [4]. Each $\mathrm{RB}$ consists of successive sub-carriers over a certain number of OFDMA symbols. Most of Radio Resources Management (RRM) schemes are compatible with OFDMA [5].

RRM is a challenging task because radio resources such as frequency and spectrum are limited and rapidly changing. RRM in LTE networks involves different technologies to cope with the increasing demand for cellular data services, making it complicated and difficult to understand and develop. Thus, the designed RRM scheme must be flexible while keeping its complexity low. TCP/IP stack layers have strict boundaries to keep their data, thus requiring sophisticated cross-layer optimization to access the stack layers data and collect radio environmental information. In addition, the coexistence of numerous radio access technologies necessitates the deployment of multiple RRM modules. These modules communicate with the radio environment and other terminals' modules to share the selected configuration of transmission parameters. In such a heterogeneous network, it is difficult to assign radio RBs to each user to satisfy QoS requirements such as packet loss and delay. Multi-path channel fading is another problem in LTE deployment. Therefore, a sophisticated RRM architecture is needed to manage spectrum allocation. Cochannel deployment is used in practice where all cells share the available spectrum with the ability to change frequency. Interference is another challenge when the spectrum is shared between macro and femto cells as in LTE-A networks [6], [7].

Joint Radio Resource Management (JRRM) [8] is one of the proposed solutions for RRM in LTE. This solution allows coexistence of multiple Radio Access Technologies (RATs) and the integration of LTE and other wireless network standards. It is based on reinforcement learning for channel allocation [9]. JRRM employs a dedicated agent in each cell to distribute users among different technologies. The focus of this solution is only on channel allocation without considering other transmission parameters optimization. Another solution is Multi Radio Resource Management (MRRM) [10] which consists of radio and mobility managers. These managers allocate channels, manage handover, and optimize load-balancing over different networks. MRRM incorporates resource management at terminal and network levels. Dynamic fractional frequency reuse is exploited for RRM in LTE to 
overcome the interference problem [11], [12]. It uses different frequency reuse factors based on the distance between the terminal and the center of the cell. High reuse factors are assigned to the terminals close to the center as they experience less interference, and vice versa. This scheme adjusts the transmission power of the terminals based on the interference information received from the downlink. All of the abovementioned schemes are based on predetermined algorithms for channel allocation. However, less attention is given to QoS support or network performance metrics, such as throughput.

The use of cognitive radio [13] in RRM can enrich the performance of LTE networks by providing environmentawareness and real-time decision-making for tuning transmission parameters [14]. Cognitive Radio Resource Management (CRRM) can be implemented at the terminal or network level. In LTE networks, it can be exploited for RBs allocation, power allocation, modulation adaptation in order to improve throughput and limit interference. These objectives are subjected to QoS requirements, user location, network operators and network policies. The nature of LTE technology makes it easy to integrate with cognitive solutions for RRM. In this paper, we propose CRRM scheme that is integrated with our cognitive based architecture (CogWnet) proposed in [15] for RRM in LTE networks. It also provides support for QoS requirements by maximizing throughput and interference mitigation using intelligent RBs assignment. Cross-layer optimization is used in the architecture to facilitate environment awareness and transmission parameters configuration. A dedicated communication interface is utilized to gather spectrum information from physical and MAC layers to be used later for decisionmaking. Bandwidth, modulation, and transmission power are adjusted to satisfy the QoS requirements and adapt to environment conditions. Learning mechanism is introduced to reduce complexity, expedite the adaptation process and improve the decision-making quality.

The rest of the paper is organized as follows. Section II presents the constrained optimization model to maximize throughput and minimize interference. Section III describes CogWnet architecture while Section IV presents the architecture of the LTE system integrated with CogWnet. It also shows how the learning mechanism is embedded in the system. Section V presents an extensive evaluation of our scheme and finally, Section VI concludes the paper and provides future directions.

\section{Constrained Throughrut AND Interference MANAGEMENT}

Throughput is a fundamental target performance metric for any spectrum optimization framework to keep at an ultimate level. Throughput threshold is used to determine the minimum acceptable throughput value that can meet QoS requirements. In order to increase throughput, the best configuration for transmission parameters is to increase the modulation index which will increase the number of bits/symbols. However, this increase has to be tied to the environment changes reflected by Bit Error Rate (BER) levels and application demands. We consider a network model to formulate throughput maximization. The model employs utility based optimization, where a utility function is specified for the total throughput for users assigned to different RBs. The utility function maps the network resources a user utilizes into a real number. Utility based optimization balances the efficiency and fairness. In this model, we consider a set of users $U=\{x: x=1, \ldots . M-1\}$ served by the network. Another set of frequency sub-carriers $F C=\{y: y=1, \ldots K-1\}$ represents the available subcarriers in the network. The throughput that can be achieved by a user $x$ assigned to sub-carrier $y$ is,

$$
T_{(x, y)}=\left(1-P_{e}\right) N_{c} M_{i} C_{r}
$$

where $P_{e}$ is the Packet Error Rate (PER), $N_{c}$ is the number of sub-carriers, $M_{i}$ is the modulation index and $C_{r}$ is the coding rate. The total throughput in which the user experiences a service is,

$$
T_{\text {all }}=\sum_{y}\left[a_{(x, y)} * T_{(x, y)}\right]
$$

where $a_{(x, y)}$ is an assignment indicator for the sub-carrier. If $a_{(x, y)}=1$ then the sub-carrier is assigned to the user and $a_{(x, y)}=0$ otherwise. The utility function selected to capture the user satisfaction about the assigned configuration is,

$$
U_{i}=0.16+0.8 \ln \left(T_{\text {all }}-0.3\right)[16]
$$

The target of the system configuration is to maximize this utility while maintaining the following constraint: $T_{\text {all }} \geq T_{\min }$ where $T_{\min }$ represents the threshold of minimum throughput to satisfy application needs.

Interference is another essential performance metric to consider especially in such heterogeneous network. In LTE context, mutual interference occurs between base stations (eNBs) contending to utilize the available radio RBs. Therefore, the RBs assignment must take place in a way that limit this interference effect and maximize the throughput of the eNBs. We consider an LTE network model to mitigate interference based on Signal to Interference and Noise Ratio (SINR) measurement. This model has multiple eNBs that cover various Hexagonal cells. The available spectrum is divided into a set of RBs. Each mobile terminal measures the reference symbol received power (RSRPs) for itself, associated eNB, and neighboring eNBs for all the RBs. The SINR is calculated by taking the average of these RSRP measurements. The SINR is recorded for all the RBs used by each eNB. As the network conditions such as network load, noise, and PER are changing, periodic updates between adjacent eNBs are exchanged. These updates include RSRPs measurements related to the eNB itself and its neighbors. Note that not all the RBs experience the same network conditions. The eNB that the terminal is associated with is denoted by $\mathrm{eNB}(\mathrm{w})$.

SINR over each RB is estimated according to the frequency used by the eNB, the power received by the terminal, and the load factors measurements at each eNB. The SINR of eNB(w) 
link is,

$$
S I N R_{e N B(w)}=\frac{P_{w} f_{w}}{\sum_{a \in S^{(w)} s r b} P_{a} f_{a} L_{a} L_{w}+n_{0}}
$$

where $P_{w}$ and $P_{a}$ are the powers received by the terminal from $\mathrm{eNB}(\mathrm{w})$ and other eNBs respectively. $f_{w}$ and $f_{a}$ are the frequencies of the RBs used by $\mathrm{eNB}(\mathrm{w})$ and other eNBs respectively. $L_{w}$ and $L_{a}$ are the load factors for eNB(w) and neighboring eNBs respectively and $n_{0}$ is the noise. $S^{(w)} s r b$ is the set of neighboring eNBs that have used the resource block $s r b$. The SINR of others eNBs' links is,

$$
S I N R_{e N B(a)}=\frac{P_{a} f_{a}}{P_{w} f_{w} L_{w}+\sum_{b \in S^{(w)} s r b} P_{b} f_{b} L_{a} L_{b}+n_{0}}
$$

where $S^{(w)} s r b$ is the set of the rest eNBs that use the resource block $s r b$.

For RB allocation, $S I N R_{\text {target }}$ is selected based on the network status to be the reference for $S I N R_{e N B(w)}$ and $S I N R_{e N B(a)}$ to compare with. SIN $R_{\text {target }}$ is updated periodically when the system acquires the environmental information based on the application type and the network conditions. This enhances the decision made for spectrum assignment. The decision about the RB assignment is determined by the following,

$$
\begin{aligned}
& s r b_{d e c 1}=S I N R_{e N B(w)}-S I N R_{\text {target }} \\
& s r b_{d e c 2}=S I N R_{e N B(a)}-S I N R_{\text {target }}
\end{aligned}
$$

If $s r b_{d e c 1} \geq 0$ and all the results of (7) are greater than or equal zero as (7) is applied for multiple eNBs. Then, the RB is selected. Note that SINR is checked for both $\mathrm{eNB}(\mathrm{w})$ and other neighbors to make the selection for the RB that was not used before by other eNBs, or used by the furthest and least number of eNBs.

\section{CogWnet: Cognitive Architecture fOR RRM}

CogWnet is an awareness-based architecture designed for cognitive radio resource management [15]. It aims to provide an abstraction of easy development and cohesive deployment of cognitive elements. It does not only target spectrum allocation, but also uses environment-awareness to optimize the transmission parameters of TCP/IP stack layers such as transmission power, modulation order, bandwidth, etc. CogWnet is a 'real' cognitive system that enables technologies to demonstrate cognitive resource management capabilities. A real cognitive system means that the system is aware of its environment and uses the environmental input to adapt to variations in the network conditions while accounting for the QoS requirements. CogWnet consists of the following layers: communication layer, which consists of interfaces and channels that exchange control signals to collect application requirements and channel information. The decision-making layer is the core of CogWnet as it accounts for receiving the sensory input from the communication layer and applies optimization algorithms to select the most suitable transmission parameters that improve the cognitive network performance. It consists of two components: repository and parameter mapper. The third layer is the policy layer which is required to enforce stakeholders and the operator's regulations, whether they are static or dynamic based on the geographical location. Trigger manager is the ring that connects the policy layer with the decision-making layer. Fig. 1 shows a high level architecture of CogWnet. CogWnet works in a distributed manner which means that it is installed in each base station in the LTE network. We integrated the above model for interference and throughput optimization with the decision-making layer in CogWnet. The parameters involved in the optimization executed by CogWnet to fulfill the model optimization goals are SINR, modulation index, PER, network load, transmission power and bandwidth. Learning modules also added to the decision-making layer in CogWnet to expedite and enhance the decision made for parameters adaptation.

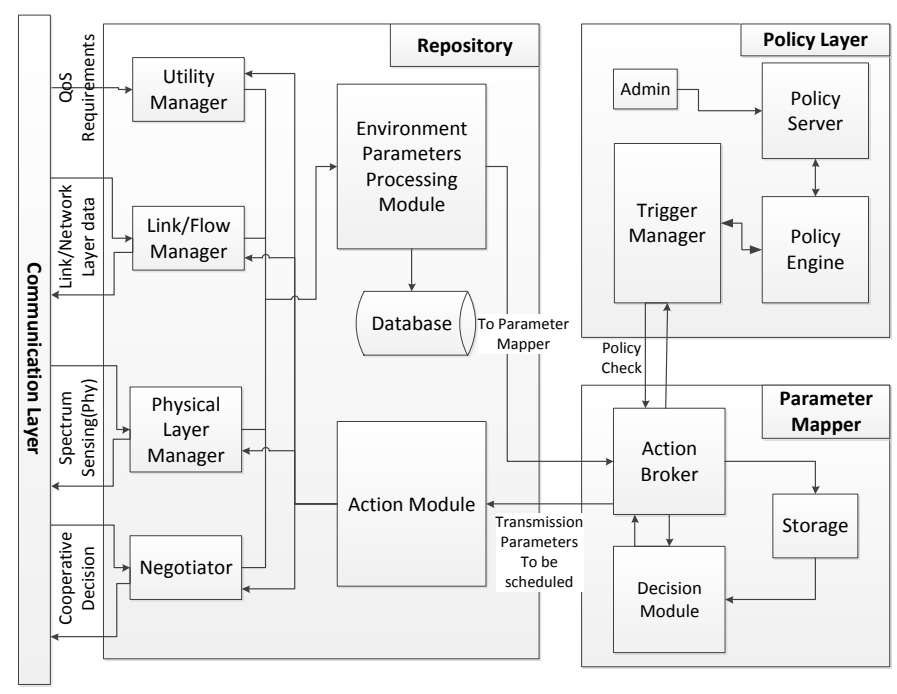

Fig. 1. CogWnet architecture

\section{Cognitive Resource Management Scheme for LTE}

This section describes our cognition-based scheme of RRM for LTE networks, starting with the design goals. The integration of CogWnet and LTE along with the interfaces exploited to facilitate the communication between the two entities are presented. We conclude this section with the overall system functionality.

The main goals of our cognitive RRM scheme are to maximize throughput, minimize interference between eNBs, improve decision-making to support QoS requirements, and expedite the optimization process through learning. Therefore, we have integrated our constrained model for throughput and interference with our cognitive architecture (CogWnet), that allocates channels that meet the QoS requirements. SINR, 
PER, number of RBs, traffic load and cell load are the environmental parameters our scheme exploits. These parameters with optimization algorithms from CogWnet are used to determine the optimal transmission parameters which include transmission power, RBs allocation, modulation index, and coding rate. To rate the performance of our scheme, effective capacity [17], throughput and user satisfaction probability are used as performance metrics. It is a link-layer capability that specifies the maximum constant arrival rate the system can support subjected to a given QoS requirement. In addition, learning modules are embedded into CogWnet architecture to improve the quality of decision-making and reduce complexity. The integration of our RRM scheme and LTE is presented below.

\section{A. Integration of LTE and Cognitive RRM Architecture}

Fig. 2 presents the integration of LTE components and our RRM scheme. The LTE components comprise one LTE UE

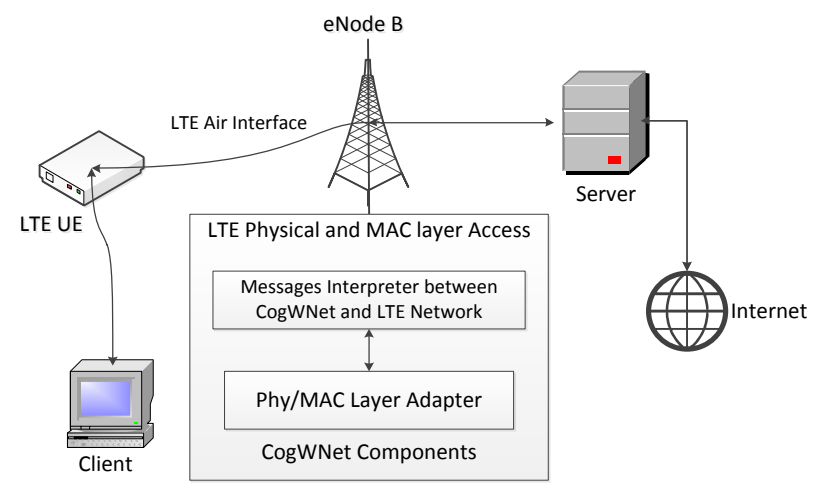

Fig. 2. Integration of LTE and CogWnet architecture

and one LTE eNB. LTE UE is the terminal equipment that includes all user activities and is compatible with the 3GPP standard. LTE eNB represents the network base station that communicates with LTE UE. In addition, it has the implementation of CogWnet architecture which executes RRM functions and controls the network activities. The communication interface between CogWnet and the LTE platform consists of two components: an interpreter and PHY/MAC layer adapter. The interpreter is implemented in the eNB to interpret CogWnet messages without changing LTE code or specifications. The PHY/MAC-layer adapter receives input from LTE PHY/MAC-layer and modifies the parameters of these layers according to the decisions made by CogWnet. The eNB is connected to an application server on the Internet to retrieve the data requested by the LTE user. The system always monitors SINR, throughput, PER, and traffic load and reacts to changes in these parameters.

\section{B. Learning Mechanism to Enhance Cognitive RRM}

Our cognitive RRM scheme adopts Reinforcement Learning (RL) [18] to improve the quality of decision made to configure the transmission parameters. User satisfaction with a service is used as a metric to represent the quality of the decision made by the cognitive engine and this should reflect the goals and the needs of the system. Throughput and interference are evaluated through the time and compared with thresholds as discussed in their model. If the measured interference is below the threshold and the throughput is above the threshold, then the user is satisfied. In addition, a database is considered in the design of the cognitive RRM scheme to store instant interactions and decisions affecting the radio environment. These interactions are exploited to make the system identifies situations encountered in the past and react. This makes the optimization faster as some complex optimization procedure will not be repeated again. The system will continue evolving gradually until it becomes aware of the best spectrum configuration.

The database in the repository of CogWnet has a table that records environmental parameters which represent the status of the network and the corresponding solution represented by transmission parameters. This table is updated after each optimization process. A new module is added to the repository in CogWnet as shown in Fig. 3 to perform the matching between the instant environment conditions and the table reference conditions. The flow of control in the repository takes place according to the following steps:

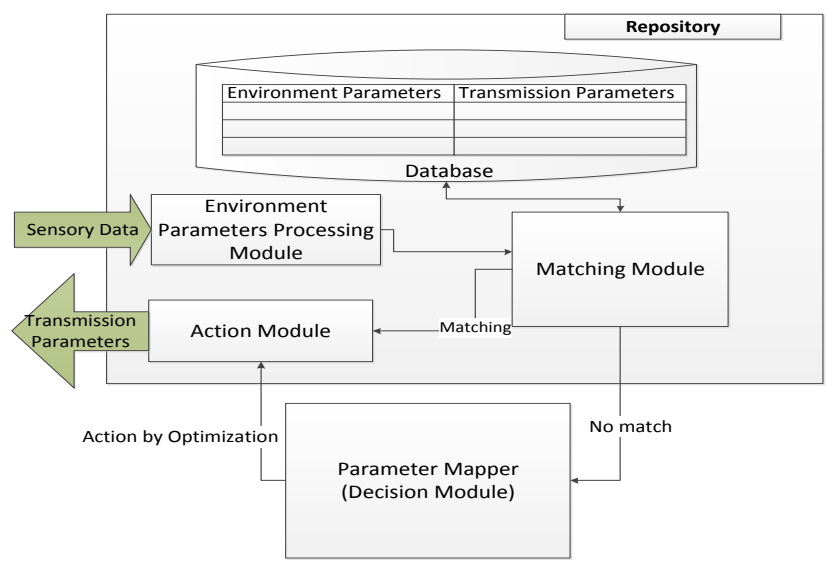

Fig. 3. Cognitive resource manager (CogWnet) with learning mechanism

1) The initial phase: Repository receives the environmental parameters from TCP/IP stack layers through access interfaces.

2) The matching phase: Matching module in the repository is triggered to check if there is a match for the current environment situation. The module will consult the database table for matching.

3) If there is a match, the corresponding configuration parameters will be passed to the action module in the repository.

4) If there is no match, the sensory information will be processed to the decision module to run the normal optimization procedure.

5) When the optimization procedure ends, the new parameters are used to configure the radio. 
6) The update phase: The new configuration is sent to the repository database and a new entry for this solution is added to the table.

\section{Cognitive RRM Architecture Functionality}

Our cognitive RRM accounts for adaptation of transmission parameters based on the environment input to achieve its overall goals. This adaptation increases LTE network efficiency by increasing throughput, improves spectrum utilization, and mitigates interference. The cognitive RRM architecture works as follows. When the system starts, CogWnet discovers the existing LTE platform. Then, it conducts periodic sensing to identify unoccupied channels. Unoccupied channels are the channels whose received interference power for certain subcarrier is less than a given threshold. When a free channel is found, CogWnet requests LTE eNB to collect information about the LTE environment such as PER, cell load, and SINR values. During the environmental information acquisition, data frames cannot be sent. Finally, CogWnet architecture acquired all the necessary information for decision-making. It runs its optimization for the proper adaptation of transmission parameters. All policies are loaded dynamically to the core of coginet.

In case the scheme detects a new service that requires a higher bit rate, the scheme considers allocation of more RBs for the associated eNB. These RBs are freed when the service is terminated. The measurement of SINR is used to manage RBs allocation and interference control. If SINR for the $\mathrm{RB}$ of the associated $\mathrm{eNB}$ is low, the scheme looks for another RB with higher SINR. PER is exploited to adapt the modulation index for throughput maximization purpose. The modulation index is increased for better throughput if the detected PER is low. On the other hand, the modulation index is decreased if the LTE link quality is poor. Transmission power is adjusted according to the measured SINR for certain RB. If the link experiences high interference, the transmission power is decreased, and vice versa. In addition, the transmission is switched to a different frequency to eliminate interference if there exsists other free channels, which can meet the applications' requirements.

\section{Performance Evaluation}

We performed extensive simulations to demonstrate the advantage of using our cognitive scheme for LTE RRM. The feasibility of cognitive RRM is represented by the optimization of network resource allocation and decision-making improvement. This is achieved by reconfiguring LTE system parameters with CogWnet. These parameters include bandwidth, coding rate, modulation index, and transmission power. The performance of our LTE RRM is evaluated using a topology which has 20 eNBs and a total bandwidth of $100 \mathrm{MHz}$. The transmission power for each eNB is uniformly divided over the RBs. The throughput of the downlink is used to evaluate the performance of this model. Table I presents a list of simulation parameters for the LTE network modeling and their default values. Fig. 4 presents the throughput achieved by our model

\begin{tabular}{|l|l|}
\hline Parameter & Value \\
\hline System Bandwidth & $100 \mathrm{MHz}$ \\
\hline RB Bandwidth & $180 \mathrm{KHz}$ \\
\hline Number of available RBs & 500 \\
\hline User distribution & Uniform \\
\hline $\begin{array}{l}\text { Background noise in } \\
\text { downlink (PN) }\end{array}$ & $-102 \mathrm{dBm}($ One RB PN /24) \\
\hline Max eNB Tx power & $20 \mathrm{~W}$ \\
\hline Multipath Fading & $3 \mathrm{GPP}-$ Case $3[19]$ \\
\hline Sub-carrier spacing $(\Delta f)$ & $15 \mathrm{KHz}$ \\
\hline Frequency reuse factor & 3 \\
\hline $\begin{array}{l}\text { Modulation } \\
\text { and coding scheme }\end{array}$ & QPSK \\
& $16-\mathrm{QAM}: 1 / 2,2 / 3,3 / 4$ \\
\hline Cell layout & Hexagonal grid \\
\hline Cell radius & $167 \mathrm{~m}$ \\
\hline Scheduler & Proportional fair queuing [20] \\
\hline
\end{tabular}

TABLE I

LTE ENVIRONMENT SIMULATION PARAMETERS

compared with typical LTE interference management scheme without cognitive capability (random scheme). The throughput is evaluated against the number of activated eNBs. The LTE interference management scheme without cognitive capability allocates radio $\mathrm{RBs}$ for each frame randomly without being aware of radio resources information. It is similar in concept to interleaved resource blocks allocation to combat the block fading channel [21]. The non-cognitive scheme has the reuse factor of 1 due to the high network load in the tested scenario. The Figure shows that our cognitive scheme is superior

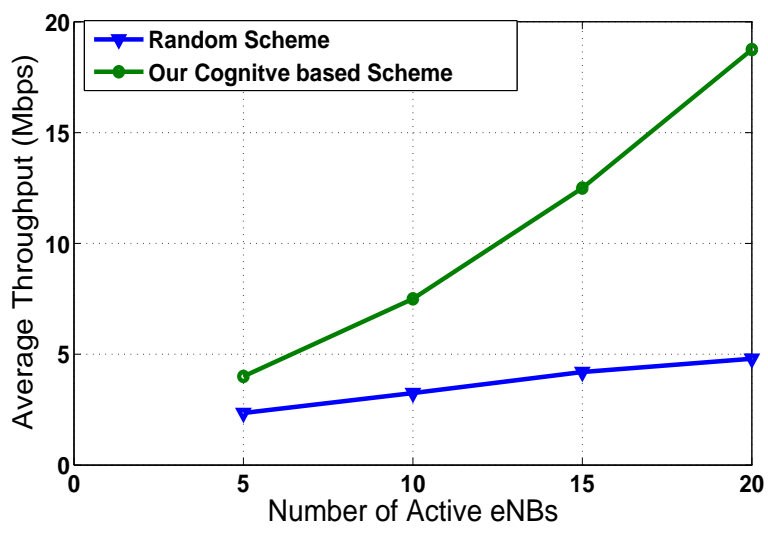

Fig. 4. Throughput per sub-carrier comparing our scheme with non-cognitive LTE scheme

compared to the non-cognitive scheme especially when the network has more active eNBs. In addition, the achieved capacity of the LTE system using our cognitive RRM scheme is compared with the one obtained by the random scheme for RRM. LTE system capacity is specified as the maximum constant arrival rate that can be supported by the system subjected to a given required QoS. Fig. 5 shows that our cognitive RRM scheme outperforms the non-cognitive scheme in terms of the achieved system capacity as a function of the traffic load.

Fig. 6 shows a comparison between our approach and 


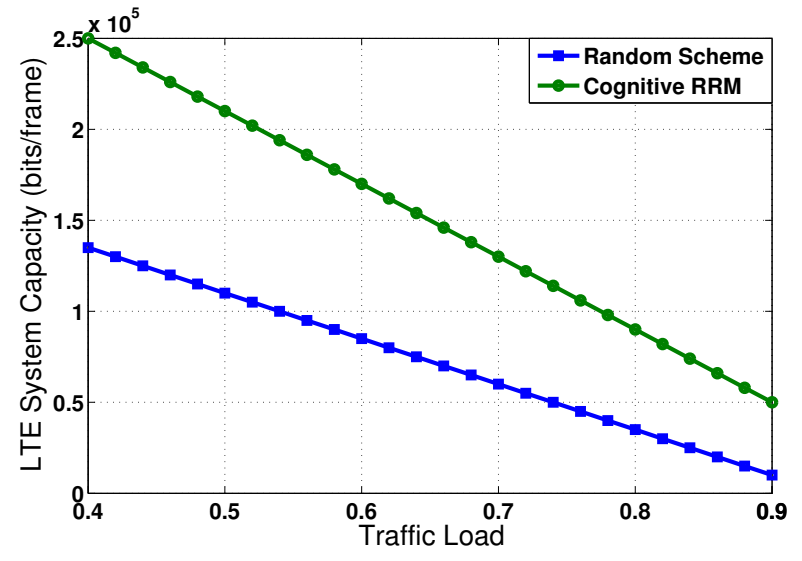

Fig. 5. LTE system capacity comparison of cognitive RRM and non-cognitive scheme

the random scheme. This comparison uses throughput as a measure for the benefit of using cognitive RRM in LTE networks. Throughput is plotted against the number of active users. The Figure shows that our approach performs much better than the random scheme as it achieves a $24 \%$ higher throughput for 30 active users per cell. The reason is that the random scheme has limited interference mitigation capability and has no throughput optimization. Overall, the cognitive solution has shown great potential for overcoming the RRM problem in LTE networks.

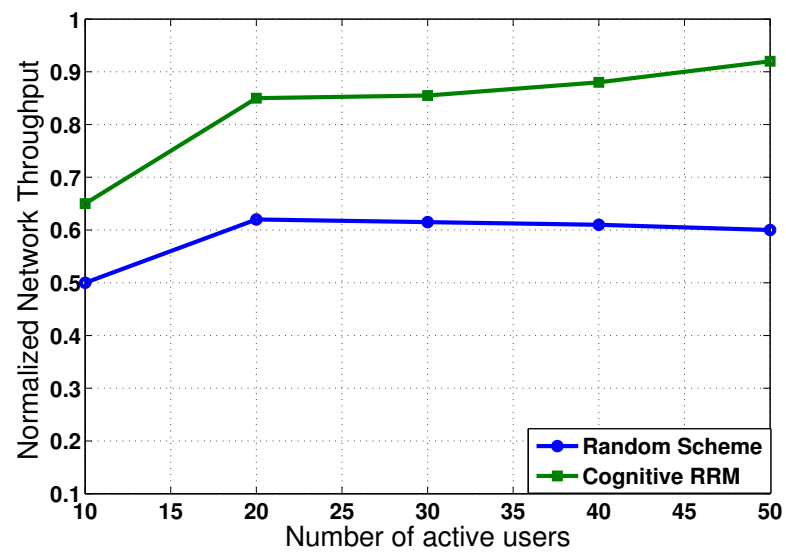

Fig. 6. LTE system normalized throughput comparison between cognitive RRM and random schemes

Another evaluation is conducted to demonstrate the quality of decision-making of our scheme for LTE system reconfiguration. This quality is represented by user satisfaction probability. Fig. 7 shows the achieved user satisfaction probability as a function of the network load. Results shows that our RL based decision-making model achieved better performance than the legacy LTE resource management, the load-balancing resource management [22] that provide access to the least loaded Radio Access Technology and the joint radio resource management scheme proposed in [23]. Complexity decrease

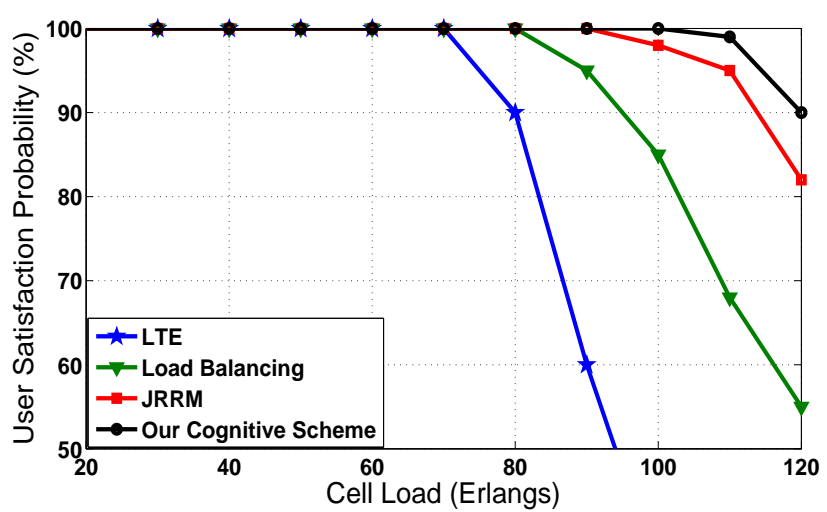

Fig. 7. User Satisfaction Probability using different RRM schemes

and how fast is the decision-making process for RRM is tested by the measure of the probability of success in matching between the current scenario and previously experienced ones. Fig. 8 depicts the evolution of successful matching probability. The evolution is tested over 5 different environment conditions. It starts from 0 as no solution was recorded and it keeps increasing as more and more solutions are recorded. The Figure shows the advantage of the learning mechanism which include saving resources, faster configuration and less complexity.

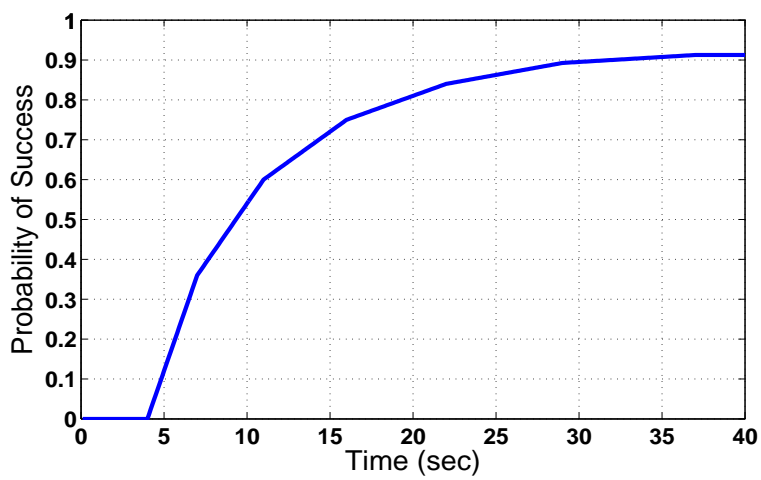

Fig. 8. The evolution of the matching probability

\section{CONCLUSION}

In this paper, we demonstrated that our integrated cognitive scheme with CogWnet is an efficient solution for RRM in LTE networks. It is a general cognitive radio architecture for RRM that is integrated with throughput and interference management model to improve efficiency in LTE networks. CogWnet is designed for high abstraction control functions and general RRM tasks. Our overall system goal was to optimize spectrum allocation, mitigate interference, maximize throughput and reduce complexity. Radio environment awareness and optimization algorithms are used to improve network efficiency and respond to changes in network conditions. Optimization starts with receiving periodic channel information. 
SINR, traffic load and PER were used to tune modulation, power, frequency, and bandwidth. In future, we would like to expand the control functions and different optimization algorithms for RRM in LTE networks.

\section{REFERENCES}

[1] E. Dahlman, H. Ekstrom, A. Furuskar, A. Kangas, J. Karlsson, M. Lundevall, and S. Parkvall, "The 3g long-term evolution - radio interface concepts and performance evaluation", in Vehicular Technology Conference, 2006, vol. 1, pp. 137-141.

[2] "Evolved universal terrestrial radio access (E-UTRA) and evolved universal terrestrial radio access network (E-UTRAN); Overall description No. 3GPP 36.300, September 2009".

[3] “3GPP, R1-050779, Texas Instruments.Throughput Evaluations in EUTRA OFDMA Downlink", 2005.

[4] S-E Elayoubi and B. Fourestie, "On frequency allocation in $3 \mathrm{~g}$ lte systems", in Personal, Indoor and Mobile Radio Communications, IEEE 17th International Symposium on, 2006, pp. 1-5.

[5] Jian Ye, Jiongkuan Hou, and S. Papavassiliou, "A comprehensive resource management framework for next generation wireless networks", Mobile Computing, IEEE Transactions on, vol. 1, no. 4, pp. 249-264, 2002.

[6] V. Chandrasekhar, J.G. Andrews, and Alan Gatherer, "Femtocell networks: a survey", Communications Magazine, IEEE, vol. 46, no. 9, pp. 59-67, 2008

[7] D. Lopez-Perez, A. Valcarce, G. de la Roche, and Jie Zhang, "Ofdma femtocells: A roadmap on interference avoidance", Communications Magazine, IEEE, vol. 47, no. 9, pp. 41-48, 2009.

[8] N. Vucevic, J. Perez-Romero, O. Sallent, and R. Agusti, "Joint radio resource management for lte-umts coexistence scenarios", in Personal, Indoor and Mobile Radio Communications, IEEE 20th International Symposium on, 2009, pp. 12-16.

[9] R. S. Sutton and A.G. Barto, Reinforcement learning: an introduction, MIT Press, Cambridge, a bradford book edition, 1998.

[10] C.M. Mueller, L. Ewe, and R. Sigle, "Signaling analysis for multi-radio management", in Wireless Communications and Networking Conference, 2009 , pp. 1-6.

[11] N. Himayat, S. Talwar, A. Rao, and R. Soni, "Interference management for $4 \mathrm{~g}$ cellular standards [wimax/lte update]", Communications Magazine, IEEE, vol. 48, no. 8, pp. 86-92, 2010.

[12] A.L. Stolyar and H. Viswanathan, "Self-organizing dynamic fractional frequency reuse for best-effort traffic through distributed inter-cell coordination", in INFOCOM IEEE, 2009, pp. 1287-1295.

[13] III Mitola, J. and Jr. Maguire, G.Q., "Cognitive radio: making software radios more personal", Personal Communications, IEEE, vol. 6, no. 4, pp. $13-18$, aug 1999.

[14] A. Saatsakis, K. Tsagkaris, D. von Hugo, M. Siebert, M. Rosenberger, and P. Demestichas, "Cognitive radio resource management for improving the efficiency of lte network segments in the wireless b3g world", in New Frontiers in Dynamic Spectrum Access Networks, DySPAN 3rd IEEE Symposium on, 2008, pp. 1-5.

[15] I. AlQerm, B. Shihada, and K. Shin, "Cogwnet: A resource management architecture for cognitive wireless networks", in To appear in proceedings of International Conference on Computer Communications and Networks, 2013.

[16] G. Song and Ye Li, "Cross-layer optimization for ofdm wireless networks-part i: theoretical framework", IEEE Transactions on Wireless Communications, vol. 4, no. 2, pp. 614-624, 2005.

[17] Dapeng Wu and R. Negi, "Effective capacity: a wireless link model for support of quality of service", Wireless Communications, IEEE Transactions on, vol. 2, no. 4, pp. 630-643, 2003.

[18] A.G. Barto R. S. Sutton, Reinforcement Learning: An Introduction, MIT Press, 1998.

[19] 3GPP TS 25.104, "Base station (BS) radio transmission and reception (FDD),release 8 (v.8.4.1)", 2008.

[20] J. Ohlhorst C. Wengerter and A. Von Elbwart, "Fairness and throughput analysis for generalized proportional fair frequency scheduling in ofdma", in IEEE 61st Vehicular Technology Conference (VTC), 2005, vol. 3, pp. 1903-1907.

[21] Sai-Weng Lei and V. K N Lau, "Performance analysis of adaptive interleaving for ofdm systems", Vehicular Technology, IEEE Transactions on, vol. 51, no. 3, pp. 435-444, 2002.
[22] J. Prez-Romero X. Gelabert and R. Agust O. Sallent, "On the suitability of load balancing principles in heterogeneous wireless access networks", in Wireless Personal Multimedia Communications Symposium (WPMC), 2005, pp. 1503-1507.

[23] O.Sallent N. Vucevic, J. Perez-Romero and R. Agusti, "Joint radio resource management for lte-umts coexistence scenarios", in IEEE 20th International Symposium on Personal, Indoor and Mobile Radio Communications (PIMRC), 2009, pp. 12-16. 